\title{
AN ALEXANDER-TYPE DUALITY FOR VALUATIONS
}

\author{
KARIM A. ADIPRASITO AND RAMAN SANYAL
}

(Communicated by Jim Haglund)

\begin{abstract}
We prove an Alexander-type duality for valuations for certain subcomplexes in the boundary of polyhedra. These strengthen and simplify results of Stanley (1974) and Miller-Reiner (2005). We give a generalization of Brion's theorem for this relative situation, and we discuss the topology of the possible subcomplexes for which the duality relation holds.
\end{abstract}

\section{INTRODUCTION}

Let $P \subset \mathbb{R}^{d}$ be a convex polytope with vertices in $\mathbb{Z}^{d}$ and let $q \in \mathbb{R}^{d}$. Viewing $q$ as a light source, let $B \subseteq \partial P$ be the collection of points in the boundary of $P$ visible from $q$ - the bright side of $P$. That is, $B$ is the set of points $p \in \partial P$ for which the open segment $(q, p)$ does not meet the relative interior of $P$. Sticking to these figurative terms, let $D$ be the closure of the set of dark points $\partial P \backslash B$. Stanley [15] showed that for integral $n \geq 1$ the function

$$
E_{P, B}(n):=\left|n \cdot(P \backslash B) \cap \mathbb{Z}^{d}\right|
$$

is the restriction of a univariate polynomial. By abuse of notation we will identify $E_{P, B}(n)$ with that polynomial. Moreover, it is shown in [15] that

$$
(-1)^{\operatorname{dim} P} E_{P, B}(-n)=\left|n \cdot(P \backslash D) \cap \mathbb{Z}^{d}\right| \text { for all } n \geq 1 \text {. }
$$

By choosing $q \in$ relint $P$, we have that $(B, D)=(\varnothing, \partial P)$ and (1) reduces to the well-known Ehrhart-Macdonald reciprocity [9]; see [3] for details. The set $B \subseteq \partial P$ is a particular case of what Ehrhart 6.7] calls a reciprocal domain, that is, a domain for which (11) holds.

For a subset $S \subset \mathbb{R}^{d+1}$, the lattice point enumerator of $S$ is the multivariate Laurent series

$$
\mathrm{F}_{S}(\mathbf{x}):=\sum_{a \in S \cap \mathbb{Z}^{d+1}} \mathbf{x}^{a}
$$

where $\mathbf{x}^{a}=x_{1}^{a_{1}} x_{2}^{a_{2}} \cdots x_{d+1}^{a_{d+1}}$. If we associate to $P$ the pointed polyhedral cone $C(P):=\operatorname{cone}(P \times\{1\})$ in $\mathbb{R}^{d+1}$ positively spanned by the points in $P \times\{1\}$, then

Received by the editors April 13, 2013.

2010 Mathematics Subject Classification. Primary 52B45, 57Q99, 52C07, 55U30.

The first author has been supported by the DFG within the research training group "Methods for Discrete Structures" (GRK1408) and by the Romanian NASR, CNCS - UEFISCDI, project PN-II-ID-PCE-2011-3-0533.

The second author has been supported by the European Research Council under the European Union's Seventh Framework Programme (FP7/2007-2013) / ERC grant agreement no 247029. 
$\mathrm{F}_{C(P)}(\mathbf{x})$ records the individual lattice points $(a, n) \in \mathbb{Z}^{d+1}$ for which $a \in n P$. Stanley [15, Prop. 8.3] actually proved the stronger result that

$$
(-1)^{\operatorname{dim} P+1} \mathrm{~F}_{C(P \backslash B)}\left(\frac{1}{\mathbf{x}}\right)=\mathrm{F}_{C(P \backslash D)}(\mathbf{x})
$$

where $\frac{1}{\mathbf{x}}=\left(\frac{1}{x_{1}}, \frac{1}{x_{1}}, \ldots, \frac{1}{x_{d+1}}\right)$.

The relation (2) holds for general rational pointed polyhedral cones $C$ but not for arbitrary subsets in the boundary of $C$. To see this, we can choose $B$ as two non-adjacent triangles in the boundary of a 3-dimensional pyramid; one can check that $B$ is not a reciprocal domain. The question of which subsets in the boundary of $C$ are reciprocal domains was investigated by Miller and Reiner [11. They showed that the conditions giving rise to reciprocal domains are topological rather than geometric in nature. Let $C \subset \mathbb{R}^{d+1}$ be a rational, pointed polyhedral cone and let $\Delta$ be a full-dimensional subcomplex of the boundary of $C$; i.e., $\Delta$ is a polyhedral complex induced by a collection of facets of $C$. Let $\Delta^{\prime}$ be the subcomplex generated by the facets $F \notin \Delta$. Their result is

Theorem 1.1 ([11, Thm. 1]). If $\Delta$ is a Cohen-Macaulay complex, then

$$
(-1)^{d+1} \mathrm{~F}_{C \backslash|\Delta|}\left(\frac{1}{\mathbf{x}}\right)=\mathrm{F}_{C \backslash\left|\Delta^{\prime}\right|}(\mathbf{x}) .
$$

The proof of Theorem 1.1 in [1] is given in terms of combinatorial commutative algebra and relies on a connection between lattice point enumerators and Hilbert series of $\mathbb{Z}^{d}$-graded modules.

In this paper we give a simple proof of (1) and (3) that generalizes to a broader class of geometric objects and to valuations other than counting lattice points (see Theorem 3.1). Our proof relies on basic facts from topological combinatorics and, as a byproduct, gives a slightly more general class of complexes for which (10) holds. Like Theorem 1.1, our results are reminiscent of Alexander duality, and we will emphasize this relation throughout.

The paper is organized as follows. In Section 2, we recall the notions of $\Lambda$-polytopes and valuations as well as (weakly) Cohen-Macaulay complexes. In Section 3 we state and prove an Alexander-duality type relation which contains Theorem 1.1 as a special case. In Section 4 we give a relative version of Brion's theorem which highlights the role played by weakly Cohen-Macaulay complexes and is of independent interest. In Section 5 we focus on the topology of full-dimensional (weakly) Cohen-Macaulay complexes in the boundary of spheres. The bright side $B$ of $P$ is homeomorphic to a ball of dimension $\operatorname{dim} P-1$ and thus Cohen-Macaulay. A natural question, which was answered affirmatively in [11, is whether there exist full-dimensional Cohen-Macaulay complexes in the boundary of polytopes that are not balls. We will extend this result and we discuss possibly counterintuitive instances for which (11) and (3) apply.

\section{2. $\Lambda$-Polytopes, valuations, and Weakly Cohen-Macaulay Complexes}

We start by setting the stage for the use of more general geometric objects and valuations, following McMullen [10]. Throughout, let $\Lambda \subset \mathbb{R}^{d}$ be a fixed, fulldimensional discrete lattice or a vector space over some subfield of $\mathbb{R}$. We denote by $\mathcal{P}=\mathcal{P}(\Lambda)$ the collection of polytopes in $\mathbb{R}^{d}$ with vertices in $\Lambda$. A $\Lambda$-valuation is a map $\varphi$ from $\mathcal{P}$ into some abelian group such that

$$
\varphi(P \cup Q)=\varphi(P)+\varphi(Q)-\varphi(P \cap Q)
$$


whenever $P \cup Q \in \mathcal{P}$ (and hence $P \cap Q \in \mathcal{P}$ ) and such that $\varphi(t+P)=\varphi(P)$ for all $t \in \Lambda$. Additionally, we require that $\varphi$ satisfies the inclusion-exclusion relation: If $P_{1}, P_{2}, \ldots, P_{k}$ are $\Lambda$-polytopes such that $P=\bigcup_{i} P_{i}$ is convex, then $\varphi$ should satisfy

$$
\varphi(P)=\sum_{i} \varphi\left(P_{i}\right)-\sum_{i<j} \varphi\left(P_{i} \cap P_{j}\right)+\cdots+(-1)^{k-1} \varphi\left(P_{1} \cap \cdots \cap P_{k}\right) .
$$

We can use the inclusion-exclusion relation to extend $\varphi$ to half-open polytopes. If $B \subset \partial P$ is the union of facets $F_{1}, F_{2}, \ldots, F_{m}$ of $P$, then

$$
\varphi(P \backslash B):=\sum_{J \subseteq[k]}(-1)^{|J|} \varphi\left(F_{J}\right) .
$$

where $F_{J}:=\bigcap\left\{F_{j}: j \in J\right\}$ and $[k]:=\{1,2, \ldots, k\}$. In particular, if $B=\partial P$, we obtain

$$
\varphi(\text { relint } P)=\sum_{F \subseteq P}(-1)^{\operatorname{dim} P-\operatorname{dim} F} \varphi(F)
$$

where the sum is over all non-empty faces $F$ of $P$. The following is the basis for our considerations.

Theorem 2.1 ([10]). Let $P$ be a $\Lambda$-polytope and $\varphi$ a $\Lambda$-valuation. Then for all $n \in \mathbb{Z}_{\geq 0}$

$$
\varphi_{P}(n):=\varphi(n P)
$$

agrees with a univariate polynomial of degree $\leq \operatorname{dim} P$ and

$$
(-1)^{\operatorname{dim} P} \varphi_{P}(-1)=\varphi(\operatorname{relint}(-P)) .
$$

A $\Lambda$-complex is a polyhedral complex $\mathcal{K}$ such that every face is a $\Lambda$-polytope. The complex is pure if all inclusion-maximal faces have the same dimension. For example, the collection of proper faces of a $\Lambda$-polytope $P$ is a pure $\Lambda$-complex, called the boundary complex $\mathcal{B}(P)$. The underlying pointset of $\mathcal{K}$ is called the support and is denoted by $|\mathcal{K}|$. Since $|\mathcal{K}|$ is the disjoint union of relatively open polytopes, we can extend $\varphi$ to $\Lambda$-complexes by setting

$$
\varphi(|\mathcal{K}|):=\sum_{F \in \mathcal{K}} \varphi(\operatorname{relint} F) .
$$

For a given face $F$ in a polyhedral complex $\mathcal{K}$, the link of $F$ in $\mathcal{K}$ is the polyhedral subcomplex

$$
\operatorname{lk}_{\mathcal{K}}(F):=\{G \in \mathcal{K}: G \cap F=\varnothing, G \cup F \subseteq H \text { for some } H \in \mathcal{K}\} .
$$

For a subcomplex $\Delta \subset \mathcal{K}$, a face $F \in \Delta$ is an interior face of $\Delta$ if $\operatorname{lk}_{\mathcal{K}}(F) \subset \Delta$ and a boundary face otherwise. The boundary of $\Delta$ is the subcomplex $\partial \Delta$ of all boundary faces. Note that for $F \notin \Delta$, we have $\operatorname{lk}_{\Delta}(F)=\varnothing \neq\{\varnothing\}$ with reduced Euler characteristic $\tilde{\chi}(\varnothing)=0$.

A pure complex $\mathcal{K}$ is weakly Cohen-Macaulay if

$$
\widetilde{H}_{i}\left(\operatorname{lk}_{\mathcal{K}}(F)\right)=0 \quad \text { for all } 0 \leq i<\operatorname{dim} \operatorname{lk}_{\mathcal{K}}(F) .
$$

for all non-empty faces $F \in \mathcal{K}$. Throughout, $\widetilde{H}_{i}(\cdot)$ refers to relative homology with $\mathbb{Z}$ coefficients. The complex $\mathcal{K}$ is Cohen-Macaulay if additionally $\widetilde{H}_{i}(\mathcal{K})=0$ for all $0 \leq i<\operatorname{dim} \mathcal{K}$. This is a stronger condition as, for instance, weakly CohenMacaulay complexes are not necessarily connected. Since $G \subseteq F$ implies $\operatorname{lk}_{\mathcal{K}}(F) \subseteq$ $\operatorname{lk}_{\mathcal{K}}(G)$, we infer that $\mathcal{K}$ is weakly Cohen-Macaulay if and only if every vertex link of $\mathcal{K}$ is Cohen-Macaulay. Munkres [13] proved that Cohen-Macaulayness of 
a complex $\mathcal{K}$ is a topological property of the support $|\mathcal{K}|$ and hence $\mathcal{K}$ is weakly Cohen-Macaulay if $\widetilde{H}_{i}(|\mathcal{K}|,|\mathcal{K}| \backslash p)=0$ for $i<\operatorname{dim} \mathcal{K}$. Note that what we define is the notion of (weakly) $\mathbb{Z}$-CM complexes as our ring of coefficients is $\mathbb{Z}$ throughout (cf. [4, Sect. 11]); however, most of our results hold for general rings of coefficients.

Finally, a pure $\Lambda$-complex $\mathcal{K}$ of dimension $d$ is a homology manifold if for every face $F$ of $\mathcal{K}$, the reduced homology of $\operatorname{lk}_{\mathcal{K}}(F)$ is identically zero or if

$$
\widetilde{H}_{i}\left(\operatorname{lk}_{\mathcal{K}}(F)\right) \cong \widetilde{H}_{i}\left(S^{d-\operatorname{dim} F+1}\right)
$$

for all $i$. In particular, if $|\mathcal{K}|$ is a manifold, then $\mathcal{K}$ is a homology manifold, and every homology manifold is weakly CM.

\section{An Alexander-type Duality}

In this section we prove Alexander-type duality relations for $\Lambda$-valuations that relate complementary complexes $\Delta$ and $\Delta^{\prime}$ inside $\Lambda$-complexes.

Theorem 3.1 (Alexander-type duality for valuations). Let $\mathcal{K}$ be a d-dimensional $\Lambda$-complex such that $\mathcal{K}$ is a homology manifold with boundary and let $B \subset \partial \mathcal{K}$ be a full-dimensional, weakly Cohen-Macaulay subcomplex. Let $D$ be the closure of $\partial P \backslash B$. If $\varphi$ is a $\Lambda$-valuation, then for all $n \geq 1$

$$
(-1)^{d} \varphi_{|\mathcal{K}| \backslash|B|}(-n)=\varphi_{-(|\mathcal{K}| \backslash|D|)}(n)
$$

and

$$
\left.(-1)^{d} \varphi_{|\mathcal{K}| \backslash|B|}(0)=\varphi(\{0\})(\widetilde{\chi}(\mathcal{K})-\widetilde{\chi}(B))\right)=\varphi_{-(|\mathcal{K}| \backslash|D|)}(0) .
$$

For the proof of the theorem we need to relate the combinatorics of inclusionexclusion for the valuation $\varphi$ to the topology of $\Delta$. The main observation, captured in the following lemma, is that weakly Cohen-Macaulay $(d-1)$-complexes that are embedded into the boundary of a $d$-dimensional homology manifold are rather restricted.

Lemma 3.2. Let $\mathcal{R}$ be a $(d-1)$-dimensional homology manifold without boundary and let $\Delta \subseteq \mathcal{R}$ be a pure, weakly Cohen-Macaulay subcomplex of full dimension $d-1$. Then for every non-empty $F \in \Delta$

$$
\widetilde{H}_{k}\left(\mathrm{lk}_{\Delta}(F)\right)= \begin{cases}\mathbb{Z} & \text { if } F \text { is an interior face of dimension } d-k-2, \text { and } \\ 0 & \text { otherwise. }\end{cases}
$$

In other words, a full-dimensional, weakly Cohen-Macaulay subcomplex of a homology manifold is again a homology manifold.

Proof. The link $\mathrm{lk}_{\Delta}(F)$ is a subcomplex of $L=\mathrm{lk}_{\mathcal{R}}(F)$, which has the homology of a $k$-sphere. Thus, if $F$ is an interior face of $\Delta$, then $\operatorname{lk}_{\Delta}(F)=L$ and $\widetilde{H}_{i}\left(\operatorname{lk}_{\Delta}(F)\right)=$ $\widetilde{H}_{i}\left(S^{k}\right)$ for all $i$.

If $\operatorname{lk}_{\Delta}(F) \subsetneq L$ is a proper subcomplex, it is sufficient to show that $\widetilde{H}_{k}\left(\mathrm{lk}_{\Delta}(F)\right)=0$ for $k=\operatorname{dimlk}_{\Delta}(F)$, as $\Delta$ is weakly Cohen-Macaulay. For this observe that $|L| \backslash\left|\mathrm{lk}_{\Delta}(F)\right|$ is non-empty. By Alexander duality for homology spheres [12, $\S 72]$, we obtain

$$
0=\widetilde{H}_{-1}\left(|L| \backslash\left|\mathrm{lk}_{\Delta}(F)\right|\right)=\widetilde{H}_{k}\left(\mathrm{lk}_{\Delta}(F)\right) .
$$

Alternatively, it is sufficient to show that $\mathrm{lk}_{\Delta}(F)$ is homotopic to a subcomplex of dimension $k-1$. To see this, note that $\operatorname{lk}_{\Delta}(F)$ is a full-dimensional subcomplex of 
the $k$-dimensional homology manifold $L$. Thus, $\mathrm{lk}_{\Delta}(F)$ has a free face and, using Whitehead's language of cellular collapses [16, $\mathrm{lk}_{\Delta}(F)$ collapses to a subcomplex of its $(k-1)$-skeleton. Since a collapse in particular provides a certificate for deformation retraction, this finishes the proof.

Proof of Theorem 3.1. As a subset of $\mathbb{R}^{d},|\mathcal{K}|$ is partitioned by the relative interiors of faces $G \in \mathcal{K}$ and thus

$$
\varphi_{|\mathcal{K} \backslash \backslash B|}(n)=\sum_{G \in \mathcal{K} \backslash B} \varphi_{\operatorname{relint} G}(n) .
$$

For the case $n \neq 0$ : as $\varphi_{|\mathcal{K}|}(n)=\varphi_{n|\mathcal{K}|}(1)$, it is sufficient to prove the claim for $n=-1$. From Theorem 2.1 and (4), we obtain

$$
\begin{aligned}
(-1)^{d} \varphi_{|\mathcal{K}| \backslash|B|}(-1) & =\sum_{G \in \mathcal{K} \backslash B}(-1)^{d-\operatorname{dim} G} \varphi(-G) \\
& =\sum_{G \in \mathcal{K} \backslash B}(-1)^{d-\operatorname{dim} G} \sum_{\sigma \subseteq G \text { face }} \varphi(\operatorname{relint}(-\sigma)) \\
& =\sum_{\sigma \in \mathcal{K}} W_{\sigma} \varphi(\operatorname{relint}(-\sigma))
\end{aligned}
$$

where for a face $\sigma \in \mathcal{K}$

$$
W_{\sigma}:=(-1)^{d} \sum_{\sigma \subseteq G \in \mathcal{K} \backslash B}(-1)^{\operatorname{dim} G}=(-1)^{d-\operatorname{dim} G}\left(\widetilde{\chi}\left(\operatorname{lk}_{\mathcal{K}}(\sigma)\right)-\widetilde{\chi}\left(\operatorname{lk}_{B}(\sigma)\right)\right) .
$$

It follows from Lemma 3.2 that $W_{\sigma}=1$ if $\sigma \in \mathcal{K} \backslash D$, which proves the claim. The proof of the case $n=0$ is analogous.

Since the boundary of every $\Lambda$-polytope is a sphere, we can extend the validity of (1) to general $\Lambda$-valuations.

Corollary 3.3. Let $P \subset \mathbb{R}^{d}$ be a $\Lambda$-polytope. Let $B$ be the support of a fulldimensional, weakly $C M$ subcomplex and let $D$ be the closure of $\partial P \backslash B$. If $\varphi$ is a $\Lambda$-valuation, then

$$
(-1)^{\operatorname{dim} P} \varphi_{P \backslash B}(-n)=\varphi_{-(P \backslash D)}(n) \text { for all } n \neq 0 .
$$

This is indeed a generalization of (1), as $\varphi(S)=\left|S \cap \mathbb{Z}^{d}\right|$ is invariant under automorphisms of the lattice $\Lambda=\mathbb{Z}^{d}$. We give an example for a self-reciprocal domain, that is, $D=\mathrm{T}(B) \subset \partial P$, where $\mathrm{T}$ is an automorphism of $\Lambda$ with $\mathrm{T}(P)=P$.

Example 3.4. Let $P=[0,1]^{4}=P_{1} \times P_{2}$ be the 4-cube presented as the product of two squares $P_{1}=P_{2}=[0,1]^{2}$. The boundary of the 4-cube contains a 2-dimensional torus $T=\partial P_{1} \times \partial P_{2}$, which decomposes $\partial P$ into two solid tori $S_{1}=P_{1} \times \partial P_{2}$ and $S_{2}=\partial P_{1} \times P_{2}$. As these are 3-manifolds with boundary, both $S_{1}$ and $S_{2}$ are pure 3-dimensional weakly Cohen-Macaulay subcomplexes. The Ehrhart function for a $k$-cube is $E_{[0,1]^{k}}(n)=(n+1)^{k}$. Thus the relative Ehrhart function is

$$
E_{P, S_{1}}(n)=(n+1)^{4}-4(n+1)^{3}+4(n+1)^{2}=n^{4}-2 n^{2}+1
$$

and $(-1)^{4} E_{P, S_{1}}(-n)=E_{P, S_{2}}(n)=E_{P, S_{1}}(n)$.

Towards a proof for Theorem 1.1 let us record the following general lemma. For a polyhedral cone $C$ and a point $a \in C$, let $\sigma_{a} \subset C$ be the unique face with $a \in \operatorname{relint} \sigma_{a}$. 
Lemma 3.5. Let $C \subset \mathbb{R}^{d+1}$ be a rational $(d+1)$-dimensional cone and $\Delta \subset \mathcal{B}(C)$ an arbitrary subcomplex. Then

$$
(-1)^{d+1} \mathrm{~F}_{C \backslash|\Delta|}\left(\frac{1}{\mathbf{x}}\right)=\mathrm{F}_{\text {relint } C}(\mathbf{x})+\sum_{a \in|\Delta| \cap \mathbb{Z}^{d+1}}(-1)^{d-\operatorname{dim} \sigma_{a}} \widetilde{\chi}\left(\operatorname{lk}_{\Delta}\left(\sigma_{a}\right)\right) \mathbf{x}^{a} .
$$

Notice that $\operatorname{lk}_{\Delta}(\sigma) \subseteq \operatorname{lk}_{\mathcal{B}(C)}(\sigma) \cong S^{d-\operatorname{dim} \sigma}$. Thus, the coefficient of $\mathbf{x}^{a}$ in the equation above is the Euler characteristic of the Alexander dual of $\left|\operatorname{lk}_{\Delta}\left(\sigma_{a}\right)\right| \subset$ $S^{d-\operatorname{dim} \sigma_{a}}$.

Proof. From Ehrhart theory (cf. [15, Prop. 7.1]), we have for a rational cone $G$

$$
(-1)^{\operatorname{dim} G} \mathrm{~F}_{G}\left(\frac{1}{\mathbf{x}}\right)=\mathrm{F}_{\text {relint } G}(\mathbf{x})
$$

Thus, from

$$
\mathrm{F}_{C \backslash|\Delta|}(\mathbf{x})=\mathrm{F}_{C}(\mathbf{x})-\sum_{G \in \Delta} \mathrm{F}_{\operatorname{relint} G}(\mathbf{x})
$$

we obtain

$$
(-1)^{d+1} \mathrm{~F}_{C \backslash|\Delta|}\left(\frac{1}{\mathbf{x}}\right)=\mathrm{F}_{\text {relint } C}(\mathbf{x})+\sum_{G \in \Delta}(-1)^{d-\operatorname{dim} G} \mathrm{~F}_{G}(\mathbf{x})
$$

which shows that the right-hand side is supported on $\operatorname{relint}(C) \cup|\Delta|$. Now for $a \in|\Delta| \cap \mathbb{Z}^{d+1}$, the coefficient of $\mathbf{x}^{a}$ on the right-hand side is

$$
\begin{aligned}
(-1)^{d+1} \sum_{\sigma_{a} \subseteq G \in \Delta}(-1)^{\operatorname{dim} G} & =(-1)^{d-\operatorname{dim} \sigma_{a}} \sum_{\bar{G} \in \mathrm{lk}_{\Delta}\left(\sigma_{a}\right)}(-1)^{\operatorname{dim} \bar{G}} \\
& =(-1)^{d-\operatorname{dim} \sigma_{a}} \widetilde{\chi}\left(\operatorname{lk}_{\Delta}\left(\sigma_{a}\right)\right),
\end{aligned}
$$

which proves the claim.

Proof of Theorem 1.1. If $\Delta$ is Cohen-Macaulay, then for every face $F \in \Delta$, the link $\mathrm{lk}_{\Delta}(F)$ has the reduced Euler characteristic of a $(d-1-\operatorname{dim} F)$-sphere if $F$ is interior and the reduced Euler characteristic of a point otherwise. Together with Lemma 3.5 this gives us

$$
(-1)^{d+1} \mathrm{~F}_{C \backslash \backslash \Delta \mid}\left(\frac{1}{\mathbf{x}}\right)=\mathrm{F}_{\text {relint } C}(\mathbf{x})+\sum_{a \in\left(|\Delta| \backslash\left|\Delta^{\prime}\right|\right) \cap \mathbb{Z}^{d+1}} \mathbf{x}^{a} .
$$

\section{A relative Brion theorem}

In this section we give a version of Brion's theorem [5] (see also [2]) suitable in the presence of a forbidden subcomplex. To make our results more transparent, let us start with the classical Brianchon-Gram relation for polytopes and an interesting complementary version. For a subset $S \subseteq \mathbb{R}^{d}$, let us denote by $[S]: \mathbb{R}^{d} \rightarrow\{0,1\}$ the indicator function. Note that $[S \cap T]=[S] \cdot[T]$.

Let $C=\left\{x \in \mathbb{R}^{d+1}:\left\langle a_{i}, x\right\rangle \leq 0\right.$ for $\left.i=1,2, \ldots, m\right\}$ be a polyhedral cone. For a non-empty face $F \subseteq C$, let $I(F)=\left\{i \in[m]:\left\langle a_{i}, x\right\rangle=0\right.$ for all $\left.x \in F\right\}$ and define the tangent cone of $C$ at $F$ as

$$
T_{C}(F):=\left\{x \in \mathbb{R}^{d}:\left\langle a_{i}, x\right\rangle \leq 0 \text { for } i \in I(F)\right\} .
$$

Lemma 4.1. Let $C \subset \mathbb{R}^{d+1}$ be a full-dimensional polyhedral cone. Then

$$
\sum_{\varnothing \neq F \subseteq C}(-1)^{\operatorname{dim} F}\left[T_{C}(F)\right]=(-1)^{d+1}[\operatorname{int}(-C)] .
$$


Proof. If $p \in \operatorname{int}(-C)$, then $p \in T_{C}(F)$ if and only if $F=C$. For $p \in \mathbb{R}^{d+1} \backslash \operatorname{int}(-C)$, let $J=\left\{i \in[m]:\left\langle a_{i}, p\right\rangle \leq 0\right\}$. Then

$$
C_{J}:=\left\{x \in \mathbb{R}^{d+1}:\left\langle a_{i}, x\right\rangle \leq 0 \text { for } i \in J\right\}
$$

is the product of a linear space and a pointed polyhedral cone and thus has Euler characteristic $\chi\left(C_{J}\right)=0$. Moreover, by sending the point $p \in C_{J}$ to infinity, the faces of $C_{J}$ are exactly those faces $F \subseteq C$ for which $p \in T_{C}(F)$ and the left-hand side of the stated equation computes the Euler characteristic of $C_{J}$.

From the previous lemma we can deduce the usual Brianchon-Gram relation (see, for example, [1, Sect. VIII.4]). If $P \subset \mathbb{R}^{d}$ is a polytope and $F \subseteq P$ a face, then the tangent cone of $P$ at $F$ is defined as $T_{P}(F):=q_{F}+\operatorname{cone}\left(P-q_{F}\right)$, where $q_{F} \in$ relint $F$. In particular, we have $T_{P}(P)=\mathbb{R}^{d}$ and $T_{P}(\varnothing)=P$. This is analogous to the definition of tangent cones for polyhedral cones above in the sense that $T_{C(P)} C(F)=C\left(T_{P}(F)\right)$.

Corollary 4.2 (Brianchon-Gram relation). If $P \subset \mathbb{R}^{d}$ is a polytope, then

$$
[P]=\sum_{\varnothing \neq F \subseteq P}(-1)^{\operatorname{dim} F}\left[T_{P}(F)\right] .
$$

Proof. Let $C=C(P) \subset \mathbb{R}^{d+1}$ be the cone associated to $P$. Let $H=\mathbb{R}^{d} \times\{1\}$. Then the $(k+1)$-faces $\hat{F}$ of $C$ bijectively correspond to $k$-faces under $F=\hat{F} \cap H$. With the appropriate identifications, $[P]=[C] \cdot[H]$ and, in particular, $\left[T_{P}(F)\right]=\left[T_{C}(\hat{F})\right] \cdot[H]$. Since $H \cap \operatorname{int}(-C)=\varnothing$, Lemma 4.1 yields

$$
[P]=[H] \cdot\left[T_{C}(0)\right]=[H] \sum_{\{0\} \subsetneq \hat{F} \subseteq C}(-1)^{\operatorname{dim} \hat{F}-1}\left[T_{C}(\hat{F})\right]=\sum_{\varnothing \neq F \subseteq P}(-1)^{\operatorname{dim} F}\left[T_{P}(F)\right],
$$

which proves the claim.

We also get a complementary version as follows. For every face $F \subseteq P$, the tangent cone is of the form $T_{P}(F)=\operatorname{aff}(F)+C_{P}(F)$ where aff $(F)$ is the affine hull of $F$ and $C_{P}(F)$ is the unique cone contained in aff $(F)^{\perp}$. Let us define the inverted tangent cone as $T_{P}^{-1}(F):=\operatorname{aff}(F)-C_{P}(F)$.

Corollary 4.3. Let $P \subset \mathbb{R}^{d}$ be a full-dimensional polytope. Then

$$
(-1)^{d}[\operatorname{relint}(P)]=\sum_{\varnothing \neq F \subseteq P}(-1)^{\operatorname{dim} F}\left[T_{P}^{-1}(F)\right] .
$$

Proof. Let $P=\left\{x:\left\langle a_{i}, x\right\rangle \leq b_{i}\right.$ for $\left.i \in[m]\right\}$. For a non-empty face $F \subseteq P$, the inverted tangent cone is given by

$$
T_{P}^{-1}(F)=\left\{x \in \mathbb{R}^{d}:\left\langle a_{i}, x\right\rangle-b_{i} \geq 0 \text { for } i \in I(F)\right\} .
$$

Now consider $C=C(-P)=\left\{(x, t): t \geq 0,\left\langle-a_{i}, x\right\rangle-b_{i} t \leq 0, i \in[m]\right\}$ and $H=\mathbb{R}^{d} \times\{-1\}$. Then, with appropriate identifications, $\operatorname{relint}(-C) \cap H=\operatorname{relint}(P)$ and $T_{C}(\hat{F}) \cap H=T_{P}^{-1}(F)$. Lemma 4.1 now yields the result.

For dealing with forbidden subcomplexes, we will also need the following relative versions of the two Brianchon-Gram relations given in Corollaries 4.2 and 4.3. If $\Delta \subseteq \mathcal{B}(P)$ is a full-dimensional subcomplex of the boundary, then it induces a subcomplex $\Delta_{F} \subseteq \mathcal{B}\left(T_{P}(F)\right)$ in the tangent cone of every face $F \subsetneq P$. This subcomplex is pure of dimension $d-1$ or empty. We write $T_{P, \Delta}(F)=T_{P}(F) \backslash\left|\Delta_{F}\right|$ 
for the tangent cone minus the faces induced by $\Delta$, and $T_{P, \Delta}^{-1}(F)$ for the analogously defined relative inverted tangent cone.

Lemma 4.4. Let $P \subset \mathbb{R}^{d}$ be a d-polytope and $\Delta \subseteq \mathcal{B}(P)$ a full-dimensional subcomplex. Let $\Delta^{\prime} \subseteq \mathcal{B}(P)$ be the subcomplex spanned by the facets not contained in $\Delta$. Then

$$
[P \backslash|\Delta|]=\sum_{F}(-1)^{\operatorname{dim} F}\left[T_{P, \Delta}(F)\right]
$$

and

$$
(-1)^{\operatorname{dim} P}\left[P \backslash\left|\Delta^{\prime}\right|\right]=\sum_{F}(-1)^{\operatorname{dim} F}\left[T_{P, \Delta}^{-1}(F)\right]
$$

where the sums are over all non-empty faces $F \subseteq P$.

Proof. We prove only the first statement as the proof of the second relation is analogous. Let $p \in \mathbb{R}^{d}$ be an arbitrary point. If $p$ is not contained in the affine span of any face of $\Delta$, then $\left[T_{P, \Delta}(F)\right](p)=\left[T_{P}(F)\right](p)$ for all non-empty faces $F \subseteq P$ and the identity is Corollary 4.2. Thus we may assume that $p$ is contained in some hyperplane spanned by a facet in $\Delta$.

If $p \in P$, then the unique face $F \subseteq P$ containing $p$ in the relative interior is a face of $\Delta$. In this case $p \in T_{P, \Delta}(G)$ if and only if $G$ and $F$ are contained in a common face of $\Delta$. That is, if $D$ is contained in the closed star $\operatorname{st}_{\Delta}(F):=\{G \in$ $\Delta: F \cup G \subseteq H$ for some $H \in \Delta\}$ of $F$ in $\Delta$. The right-hand side of (5) evaluated at $p$ can be written as

$$
\sum_{F \in \mathcal{B}(P) \backslash\{\varnothing\}}(-1)^{\operatorname{dim} F}-\sum_{D \in \mathrm{st}_{\Delta}(F) \backslash\{\varnothing\}}(-1)^{\operatorname{dim} D} .
$$

This is the difference of the unreduced Euler characteristics of two contractible complexes and therefore $0=1-1$.

If $p \in \mathbb{R}^{d} \backslash P$, let $F_{1}, \ldots, F_{k} \subseteq P$ be the $(d-1)$-dimensional faces of $\Delta$ for which $p$ is contained in the affine hyperplane $H_{i}:=\operatorname{aff}\left(F_{i}\right)$ spanned by $F_{i}$. We have to show that

$$
\sum\left\{(-1)^{\operatorname{dim} G}: p \in T_{P}(G) \text { and } G \subseteq F_{i} \text { for some } i=1, \ldots, k\right\}=0,
$$

as this is the collection of terms missing from the usual Brianchon-Gram relation. For $I \subseteq[k]$, let $F_{I}=\cap_{i \in I} F_{i}$ and define

$$
s_{I}:=\sum\left\{(-1)^{\operatorname{dim} G}: p \in T_{P}(G) \text { and } G \subseteq F_{I}\right\} .
$$

We can rewrite the left-hand side of (7) as

$$
\sum_{\varnothing \neq I \subseteq[k]}(-1)^{|I|-1} s_{I} .
$$

But for a fixed $I$, we have that $s_{I}$ is equal to the left-hand side of the BrianchonGram relation applied to $F_{I}$ and a point $p \notin F_{I}$ inside aff $\left(F_{I}\right)$. Thus $s_{I}=0$.

We can now state our generalization of Brion's theorem.

Theorem 4.5 (Relative Brion theorem). Let $P \subset \mathbb{R}^{d}$ be a full-dimensional polytope with vertices $v_{1}, v_{2}, \ldots, v_{n} \in \mathbb{Z}^{d}$. Let $\Delta \subseteq \mathcal{B}(P)$ be a pure and d-dimensional weakly 
Cohen-Macaulay subcomplex and let $\Delta^{\prime} \subseteq \mathcal{B}(P)$ be the subcomplex generated by the facets of $P$ not contained in $\Delta$. Then

$$
\mathrm{F}_{P \backslash|\Delta|}(\mathbf{x})=\mathrm{F}_{T_{P, \Delta}\left(v_{1}\right)}(\mathbf{x})+\mathrm{F}_{T_{P, \Delta}\left(v_{2}\right)}(\mathbf{x})+\cdots+\mathrm{F}_{T_{P, \Delta}\left(v_{n}\right)}(\mathbf{x})
$$

and

$$
(-1)^{d} \mathrm{~F}_{P \backslash|\Delta|}\left(\frac{1}{\mathbf{x}}\right)=\mathrm{F}_{-\left(P \backslash\left|\Delta^{\prime}\right|\right)}(\mathbf{x}) .
$$

Proof. The first statement follows from the same consideration as in [2]: Observe that for $S \subset \mathbb{R}^{d}$, we have $\mathrm{F}_{S}(x)=\sum_{a \in \mathbb{Z}^{d}}[S](a) \mathbf{x}^{a}$, and Lemma 4.4 yields

$$
\mathrm{F}_{P \backslash|\Delta|}(\mathbf{x})=\sum_{F}(-1)^{\operatorname{dim} F} \mathrm{~F}_{T_{P, \Delta}(F)}(\mathbf{x})
$$

where the sum is over all non-empty faces $F \subseteq P$. Now if $F$ is not a vertex, the relative tangent cone $T_{P, \Delta}(F)$ is not pointed, that is, $t+T_{P, \Delta}(F)=T_{P, \Delta}(F)$ for some $t \neq 0$. On the level of lattice point enumerators, this means $\mathbf{x}^{t} \mathrm{~F}_{T_{P, \Delta}(F)}(\mathbf{x})=$ $\mathrm{F}_{T_{P, \Delta}(F)}(\mathbf{x})$ and thus $\mathrm{F}_{T_{P, \Delta}(F)}(\mathbf{x})=0$. This proves the first statement.

By the same token, we conclude from Lemma 4.4

$$
(-1)^{d} \mathrm{~F}_{P \backslash\left|\Delta^{\prime}\right|}(\mathbf{x})=\sum_{F}(-1)^{\operatorname{dim} F} \mathrm{~F}_{T_{P, \Delta}^{-1}(F)}(\mathbf{x})
$$

and thus

$$
(-1)^{d} \mathrm{~F}_{P \backslash\left|\Delta^{\prime}\right|}(\mathbf{x})=\sum_{i=1}^{n} \mathrm{~F}_{T_{P, \Delta}^{-1}\left(v_{i}\right)}(\mathbf{x}) .
$$

Let us write $T_{P, \Delta}\left(v_{i}\right)=v_{i}+C_{i} \backslash\left|\Delta_{v_{i}}\right|$ where $C_{i}$ is a rational polyhedral cone and $\Delta_{i}=\Delta_{v_{i}}$ is the subcomplex induced by $\Delta$. In particular, $\mathrm{F}_{T_{P, \Delta}\left(v_{i}\right)}(\mathbf{x})=$ $\mathbf{x}^{v_{i}} \mathrm{~F}_{C_{i} \backslash \backslash \Delta_{i} \mid}(\mathbf{x})$. Since $\Delta$ is weakly Cohen-Macaulay, we have that $\Delta_{i}$ is CohenMacaulay, and by Theorem 1.1

$$
(-1)^{d} \mathrm{~F}_{T_{P, \Delta}\left(v_{i}\right)}\left(\frac{1}{\mathbf{x}}\right)=\mathbf{x}^{-v_{i}} \mathrm{~F}_{C_{i} \backslash \backslash \Delta_{i}^{\prime} \mid}(\mathbf{x})=\mathrm{F}_{T_{-P,-\Delta^{\prime}}^{-1}\left(-v_{i}\right)}(\mathbf{x}) .
$$

For the finishing touch, we calculate

$\mathrm{F}_{P \backslash \backslash \Delta \mid}\left(\frac{1}{\mathbf{x}}\right)=\sum_{i=1}^{n} \mathrm{~F}_{T_{P, \Delta}\left(v_{i}\right)}\left(\frac{1}{\mathbf{x}}\right)=\sum_{i=1}^{n}(-1)^{d} \mathrm{~F}_{T_{-P,-\Delta^{\prime}}^{-1}\left(-v_{i}\right)}(\mathbf{x})=(-1)^{d} \mathrm{~F}_{-\left(P \backslash\left|\Delta^{\prime}\right|\right)}(\mathbf{x})$.

\section{TOPOLOGY OF RECIPROCAL DOMAINS}

Theorems 1.1 and 3.1 apply to full-dimensional (weakly) Cohen-Macaulay complexes in the boundaries of polytopes. In this section we discuss what forms these complexes can take. In [11, Miller and Reiner gave an example of a full-dimensional Cohen-Macaulay subcomplex in the boundary of a polytope that is not contractible and hence not a ball; they argued that, for instance, the Mazur manifold can occur. The purpose of this section is to generalize this remark. We refer to [14 for the basic notions of PL topology.

Theorem 5.1. Let $B$ be any $P L$ manifold of dimension $d \geq 5$ such that

(a) the natural inclusion $\pi_{1}(\partial B) \hookrightarrow \pi_{1}(B)$ is surjective, and

(b) $B$ is homologically trivial; i.e., $\widetilde{H}_{i}(B)=0$ for all $i \geq 0$.

Then there exist a $(d+1)$-polytope $P$ and a subcomplex $\widetilde{B} \subseteq \mathcal{B}(P)$ such that $\widetilde{B}$ is $P L$ homeomorphic to $B$. In particular, $\widetilde{B}$ is a full-dimensional weakly Cohen-Macaulay subcomplex of $\partial P$. 
Any homology manifold $B$ satisfying assumptions (a) and (b) is a homology ball.

Proof. By [8, Thm. 3], there is a contractible PL manifold $M$ for which $\partial M$ is $\mathrm{PL}$ homeomorphic to $\partial B$. Then the gluing of $M$ and $B$ along their boundaries is a PL-sphere $S$, since it is PL (because $B$ and $M$ are PL), simply connected (by property (a) of $B$ and the fact that $M$ is contractible) and has the homology of a sphere (since both $M$ and $B$ have the homology of a sphere); consequently, it is a PL sphere by the generalized Poincaré conjecture [17. In particular, we have that there exists a subdivision $S^{\prime}$ of $S$ that is combinatorially equivalent to the boundary complex $S^{\prime \prime}$ of a $(d+1)$-polytope $P$. The subcomplex of $S^{\prime \prime}$ corresponding to $B$ is the desired complex $\widetilde{B}$.

Corollary 5.2. Every contractible $P L d$-manifold $B$ for $d \geq 5$ can be realized, up to PL homeomorphism, as a full-dimensional weakly Cohen-Macaulay subcomplex in the boundary of a $(d+1)$-polytope.

The corollary suggests that every PL manifold satisfying (a) and (b) of Theorem 5.1 is contractible. This is not the case:

Example 5.3. Let $S$ denote a PL homology sphere that's not $S^{d}$, such as Poincaré's homology sphere, and let $\Delta$ denote any facet of $S$. Then, $B:=(S-\Delta) \times[0,1]$ is a homology ball, but homotopy equivalent to $S-\Delta$, which has $\pi_{1}(S)=\pi_{1}(S-\Delta) \neq 0$ and is consequently not contractible.

Theorem 3.1 applies more generally to subcomplexes in the boundary of homology manifolds; in this case, we are surprisingly flexible:

Theorem 5.4. Let $M$ denote any homology manifold with vanishing reduced homology. Then there exists a homology ball that contains $M$ as a full-dimensional subcomplex of its boundary.

Proof. Let $D(M, \partial M)$ denote the double of $M$ (that is, the result of gluing two manifolds PL homeomorphic to $M$ along their isomorphic boundaries). By an elementary Mayer-Vietoris-type argument, the complex $D(M, \partial M)$ is a homology manifold without boundary that is homologically equivalent to a sphere. Thus, the cone over $D(M, \partial M)$ is a homology ball, as desired.

\section{REFERENCES}

[1] A. Barvinok, A course in convexity, Graduate Studies in Mathematics, vol. 54, American Mathematical Society, Providence, RI, 2002. MR1940576 (2003j:52001)

[2] M. Beck, C. Haase, and F. Sottile, Formulas of Brion, Lawrence, and Varchenko on rational generating functions for cones, Math. Intelligencer 31 (2009), no. 1, 9-17, DOI 10.1007/s00283-008-9013-y. MR2480796 (2010i:52024)

[3] M. Beck and S. Robins, Computing the continuous discretely: Integer-point enumeration in polyhedra, Undergraduate Texts in Mathematics, Springer, New York, 2007. MR2271992 (2007h:11119)

[4] A. Björner, Topological methods, Handbook of combinatorics, Vol. 1, 2, Elsevier, Amsterdam, 1995, pp. 1819-1872. MR 1373690 (96m:52012)

[5] M. Brion, Points entiers dans les polyèdres convexes (French), Ann. Sci. École Norm. Sup. (4) 21 (1988), no. 4, 653-663. MR982338 (90d:52020)

[6] E. Ehrhart, Sur un problème de géométrie diophantienne linéaire. I. Polyèdres et réseaux (French), J. Reine Angew. Math. 226 (1967), 1-29. MR0213320 (35 \#4184)

[7] E. Ehrhart, Sur un problème de géométrie diophantienne linéaire. II. Systèmes diophantiens linéaires (French), J. Reine Angew. Math. 227 (1967), 25-49. MR0217010 (36 \#105) 
[8] M. A. Kervaire, Smooth homology spheres and their fundamental groups, Trans. Amer. Math. Soc. 144 (1969), 67-72. MR0253347 (40 \#6562)

[9] I. G. Macdonald, Polynomials associated with finite cell-complexes, J. London Math. Soc. (2) 4 (1971), 181-192. MR.0298542 (45 \#7594)

[10] P. McMullen, Valuations and Euler-type relations on certain classes of convex polytopes, Proc. London Math. Soc. (3) 35 (1977), no. 1, 113-135. MR0448239 (56 \#6548)

[11] E. Miller and V. Reiner, Reciprocal domains and Cohen-Macaulay d-complexes in $\mathbb{R}^{d}$, Electron. J. Combin. 11 (2004/06), no. 2, Note 1, 9 pp. (electronic). MR2120111 (2005k:52024)

[12] J. R. Munkres, Elements of algebraic topology, Addison-Wesley Publishing Company, Menlo Park, CA, 1984. MR755006 (85m:55001)

[13] J. R. Munkres, Topological results in combinatorics, Michigan Math. J. 31 (1984), no. 1, 113-128, DOI 10.1307/mmj/1029002969. MR.736476 (85k:13022)

[14] C. P. Rourke and B. J. Sanderson, Introduction to piecewise-linear topology: Ergebnisse der Mathematik und ihrer Grenzgebiete, Band 69, Springer-Verlag, New York, 1972. MR0350744 (50 \#3236)

[15] R. P. Stanley, Combinatorial reciprocity theorems, Advances in Math. 14 (1974), 194-253. MR.0411982 (54 \#111)

[16] J. H. C. Whitehead, Simplicial spaces, nuclei and m-groups, Proc. London Math. Soc. S2-45, no. 1, 243, DOI 10.1112/plms/s2-45.1.243. MR1576810

[17] E. C. Zeeman, The generalised Poincaré conjecture, Bull. Amer. Math. Soc. 67 (1961), 270. MR0124906 (23 \#A2215)

Institut des Hautes Études Scientifiques, Paris, France

E-mail address: adiprasito@ihes.fr, adiprssito@math.fu-berlin.de

Fachbereich Mathematik und Informatik, Freie Universität Berlin, Berlin, Germany

E-mail address: sanyal@math.fu-berlin.de 